\title{
Xylem Sap Metabolite Profile Changes During Phytostimulation of Maize by the Plant Growth-Promoting Rhizobacterium, Azospirillum lipoferum CRT1
}

\author{
Camille Rozier $^{1 *}$, Alexander Erban ${ }^{2}$, Jihane Hamzaoui ${ }^{1}$, Claire Prigent-Combaret ${ }^{1}$, Gilles Comte ${ }^{1}$, Joachim Kopka ${ }^{2}$, Sonia Czarnes ${ }^{1}$ and
} Laurent Legendre ${ }^{1}$

${ }^{1}$ CNRS, Microbial Ecology, Université de Lyon, Lyon, France

${ }^{2}$ Max Planck Institute for Molecular Plant Physiology, Am Muehlenberg 1, D-14476 Golm, Germany

\begin{abstract}
Plant Growth-Promoting Rhizobacteria (PGPR) enhances host plant growth and tolerance to biotic and abiotic stresses. Despite increased knowledge of their functional activities, reports of their impact on host metabolism and signalling networks are rare. In this study, small organic substances were analysed in the ascending xylem sap of maize plantlets that were inoculated with the PGPR Azospirillum lipoferum. In this feasibility study, xylem sap collection using a Scholander chamber was combined with metabolome analysis by Gas Chromatography-Mass Spectrometry (GC-MS) based profiling. Two genotypes of maize were investigated. Cultivar Seiddi displayed A. lipoferum-mediated increases in lateral root growth and enhanced photosynthetic potential unlike non-responsive cultivar FuturiXX. A total of 119 small organic substances were annotated in maize xylem sap. The content of 17 substances, including primary metabolites, such as sucrose, maltose, glucose, TCA cycle intermediates, amino acids, GABA and shikimate pathway metabolites, decreased in both cultivars after $A$. lipoferum inoculation and may thus reflect general effects of the maize-A. lipoferum interaction. The content of 28 additional substances, namely glucose, lactic acid, acidic intermediates of the pentose phosphate and ascorbate/aldarate pathways and defense-related hydroxycinnamic acids, specifically changed in the xylem sap of the A. lipoferum-phytostimulated cultivar Seiddi, therefore, suggesting that phytostimulation of maize by $A$. lipoferum may involve xylem-transported metabolic signalling. Glucose or other metabolites that are retrograde transported through the xylem to the shoot by transpirational pull may act as feedback signals of the root status. Such signals may stimulate leaves to enhance photosynthesis-mediated $\mathrm{C}$-assimilation that is needed to sustain $A$. lipoferum-triggered root growth. The untargeted metabolome analysis of the xylem, i.e., the xylenome, indicates that the differential interactions of the two maize cultivars Seiddi and FuturiXX with Azospirillum lipoferum could represent a feasible system for the study of the role of xylem transported signals in plant/ PGPR interactions.
\end{abstract}

Keywords: Azospirillum; Glucose signalling; Metabolite profiling; PGPR; Photosynthesis; Root-to-shoot communication; Xylem sap

\section{Introduction}

Inoculation of crop seeds with symbiotic bacteria has recently become a promising source of technologies to sustain plant productivity under reduced mineral nutrition and enhanced climatic havoc [1]. Among the many Plant Growth-Promoting Rhizobacteria (PGPR), some engage in a mutualistic relationship whereby the interaction is intimate, mostly obligate, and leads to the development of specialized plant structures such as nodules [2]. Though very efficient for nitrogen fixation, these interactions are a rarity in the plant kingdom as they concern few plant families like the Fabaceae, Casuarinaceae and Betulaceae, and few bacterial genera such as Frankia, Rhizobium, and related genera [2]. In most other cases, symbiotic plant-bacteria associations are merely cooperative. They are called associative symbioses, involve less obligate and specific relationships and concern diverse genera mostly from the Firmicute and Proteobacteria [3]. They rarely live as endophytes in the root cortex but colonize discrete spots on the surface of plant roots where they efficiently compete with other rhizosphere-associated microorganisms for the abundant and energy-rich exudates that ooze from plant roots [3]. Associative symbiotic bacteria promote better plant growth and health through direct and indirect mechanisms [4]. Among them, members of the genus Azospirillum have attracted particular attention for fieldapplications as growth-stimulators because of their innocuousness towards humans, and the environment, and because of their capacity to stimulate the growth of cereal crops [5]. A meta-analysis of field inoculation experiments of wheat conducted between 1981 and 2008 notably revealed their capacity to enhance grain and forage yields by 14 and $27 \%$, respectively, ( $n=91$ field trials) in the absence of nitrogen fertilization [6]. Yield enhancements were lowered in the presence of mineral fertilization and were not further boosted by co-inoculation with the nitrogen-fixing PGPR genus Azotobacter [6]. Though most Azospirillum isolates are equipped with a functional dinitrogen-fixing nifH gene, its contribution to host plant growth has been the subject of debate and is thought to be, at best, minor [7]. It is accepted that Azospirillum stimulates plant growth by secreting auxin type plant hormones and, in a few cases cytokinins, and gibberellins, to alter root system architecture and physiology and as a consequence, plant ability to access soil water and minerals $[4,7]$.

Increased nutrient sourcing and root function will positively feedback to increase growth of above ground parts. So far, indirect lines of evidence suggest that PGPR may affect aerial plant parts also independently of nutritional effects. The biocontrol agent Bacillus improved photochemical conversion in Solanum tuberosum and tolerance to salt, drought or heavy metals stresses [8]. PGPR produced cytokinins were found to increase host plant leaf cytokinin concentration in parallel to growth increases of this organ. Root

*Corresponding author: Rozier C, CNRS, Microbial Ecology, Université de Lyon, INRA, UMR1418, Villeurbanne, Lyon, France, Villeurbanne, France, Tel: 0033750600583; E-mail: camille.rozier@univ-lyon1.fr

Received July 07, 2016; Accepted July 26, 2016; Published July 29, 2016

Citation: Rozier C, Erban A, Hamzaoui J, Prigent-Combaret C, Comte G, et al. (2016) Xylem Sap Metabolite Profile Changes During Phytostimulation of Maize by the Plant Growth-Promoting Rhizobacterium, Azospirillum lipoferum CRT1. Metabolomics (Los Angel) 6: 182. doi:10.4172/2153-0769.1000182

Copyright: ( 2016 Rozier C, et al. This is an open-access article distributed under the terms of the Creative Commons Attribution License, which permits unrestricted use, distribution, and reproduction in any medium, provided the original author and source are credited. 
Citation: Rozier C, Erban A, Hamzaoui J, Prigent-Combaret C, Comte G, et al. (2016) Xylem Sap Metabolite Profile Changes During Phytostimulation of Maize by the Plant Growth-Promoting Rhizobacterium, Azospirillum lipoferum CRT1. Metabolomics (Los Angel) 6: 182. doi:10.4172/21530769.1000182

Page 2 of 10

inoculation with $P$. fluorescens was shown to Induce Systemic Resistance (ISR) in Arabidopsis thaliana and boosted tolerance to both root and leaf pathogens including P. syringae [9]. Accordingly, several plant defence hormones, such as nitric oxide and Abscisic Acid (ABA) which concentrations are modulated by several PGPR in plant roots, are also known to regulate plant defence gene expression both locally and at a distance (systemically) via their ability to activate plant ISR [10]. Nevertheless, studies showing direct modulation of root to shoot signalling molecules by PGPR in host plants are still missing.

In most known cases, roots communicate with shoots via soluble mineral and small organic substances carried by the up-moving xylem sap [11,12]. Nevertheless, upward transport in phloem vessels has recently been observed for larger organic substances such as mRNAs and proteins [13]. Research in xylem sap mediated transport has been slow because of the difficulty to obtain xylem sap samples devoid of phloem, or other cellular contaminants. Contamination is typically unavoidable unless sap movement is forced either by pressurizing the root surface or by depressurizing the collect site on the shoot [14]. In addition, the speed of xylem sap outflow was found to affect its constitution [15]. These shortcomings can be avoided by applying to the roots a pressure equal to the water potential of a growing leaf in a Scholander pressure chamber in order to force xylem sap to flow at physiological speed [16]. In most xylem sap metabolite studies, only targeted metabolites were analysed such as ions and hormones [17-20], phenylpropanoids or amino acids, sugars and organic acids [21,22]. So far, only one study has employed global metabolomic analyses to characterize the impact of iron deficiency on xylem sap metabolite profile [23] so that, to date, xylem sap metabolite content still remains insufficiently characterized.

In this study, we used up-to-date metabolomic tools to conduct an analysis of xylem sap metabolites during plant-PGPR interactions using the commercial PGPR strain A. lipoferum CRT1 and maize as a plant model system [24]. Two genotypes of maize that exhibit differential growth responses to A. lipoferum CRT1 were chosen to highlight single metabolite, or differential patterns of metabolites that are either associated with A. lipoferum colonization of maize roots or with growth-promotion and associated shoot physiological changes.

\section{Materials and Methods}

\section{Bacterial culture}

Inoculation assays were carried out using the PGPR strain Azospirillum lipoferum CRT1 (Fages and Mulard) according to a previously published protocol with the following modifications $[25,26]$. A total of $15 \mu \mathrm{L}$ of bacterial glycerol stock solution was spread on a solid medium $(5.75 \mathrm{~g}$ Nutrient Agar and $2 \mathrm{~g} \mathrm{NaCl}$, in $250 \mathrm{ml}$ ) and left to grow for $4 \mathrm{~d}$ at $28^{\circ} \mathrm{C}$. A single colony was selected and subjected to pre-culture and culture at $28^{\circ} \mathrm{C}$ under mild agitation $(200 \mathrm{rpm})$ in Nitrogen-free broth supplemented with $1 / 40(\mathrm{v} / \mathrm{v}) \mathrm{LB}$ medium and $0.5 \%$ biotin $(50 \mathrm{mg}$ in $50 \mathrm{~mL} \mathrm{NaOH}$ $0.1 \mathrm{~N})[27,28]$. After a first centrifugation $(10 \mathrm{~min}, 3800 \mathrm{rpm})$ bacterial cells were washed with sterile UP water, centrifuged again and resuspended in $20 \mathrm{~mL}$ sterile UP water. The adequacy of the bacterial suspension was first evaluated by PCR with the A. lipoferum CRT1specific primers F1676-Q1 (5'-ATCCCGGTGGACAAAGTGGA-3') and 1837-Q2 (5'-GGTGCTGAAGGTGGAGAACTG-3') [29]. Then, the proper mobility and the absence of contaminants were checked with light microscopy. The bacterial suspension was diluted to a concentration of $1.10^{7}$ cells $/ \mathrm{mL}$ with sterile UP water and immediately used to inoculate maize seeds.

\section{Seed inoculation}

Seeds of the maize (Zea mays subsp. mays L.) genotypes FuturiXX (RAGT) and Seiddi (Caussade-Semences) that had similar mass (between 0.3609 and $0.3780 \mathrm{~g}$ ) and shape were selected in order to reduce growth heterogeneity. Bacterial inoculation was performed at room temperature by depositing a $50 \mu \mathrm{L}$ drop of the diluted bacterial suspension on each side of the maize seed. Mock-inoculated, control, seeds were treated similarly except that the bacterial suspension was replaced by UP water. This operation was repeated three times at $1 \mathrm{~h}$ intervals. During this process, seeds were stored in the dark and laid flat on a filter paper dampened with UP sterile water and enclosed in a plastic petri dish. They were then left undisturbed at room temperature in such a setup for an additional $9 \mathrm{~h}$ before sowing.

\section{Maize cultivation}

Top soil (5-30 $\mathrm{cm}$ below the surface) from a commercial maize luvisol field was sampled at Sérézin de la Tour (Isère, France) in early spring 2015 and sieved $(<4 \mathrm{~mm})$ upon harvest. It had a clay-loam texture devoid of limestone and consisted of $26.9 \%$ sand, $38.3 \%$ loam and $34.7 \%$ clay with $324.9 \mathrm{~g}$ of water per $\mathrm{kg}$ of soil at field water holding capacity. Organic matter content was $54.3 \mathrm{~g} / \mathrm{kg}$ and Cation Exchange Capacity (CEC) was $228 \mathrm{meq} / \mathrm{Kg}$. pH (water) was equal to 7.05 and $\mathrm{pH}(\mathrm{KCl})$ was 6.28 . Sieved soil was introduced into pots $(160 \mathrm{~mm}$ high and $60 \mathrm{~mm}$ diameter) at a density of $1.2 \mathrm{~g} . \mathrm{cm}^{-3}$ at soil water holding capacity. Soil water content was adjusted to its field water holding capacity (32.49\% humidity) and immediately used for plant cultivation.

Inoculated and control seeds were sown about $0.5 \mathrm{~cm}$ below the soil surface and placed in a growth chamber at constant air humidity (60\% RH), lightning (16/8 h light/dark cycle and $815 \mu$ mol. $\mathrm{m}^{-2}$ photon density) and temperature $\left(24^{\circ} \mathrm{C}\right)$. Soil water content was adjusted daily for each individual pot to maintain them at field water holding capacity. The entire experiment was repeated 4 times successively to generate 4 independent biological replicates.

\section{Photosynthesis efficiency and chlorophyll content measurements}

After $11 \mathrm{~d}$ of culture, chlorophyll fluorescence of the second leaf (most recent mature leaf) was measured about one third down from the leaf tip with a portable photosynthesis yield analyzer (Mini-PAMII, Walz, Germany) equipped with the clip holder 2035-B. For this, maize plantlets were first adapted to darkness for $30 \mathrm{~min}$. Then the maximal Fluorescence $(\mathrm{Fm})$ was measured at $20 \mathrm{kHz}$ with a $1 \mathrm{~s}$ pulse of $6000 \mu \mathrm{mol}$ photon. $\mathrm{m}^{-2} \mathrm{~s}^{-1}$ of 'white light'. F0 was measured in parallel with a pulse of dark red light that activated PSII reaction centres only via the excitation of PSI. The maximum quantum efficiency of PSII primary photochemical conversion ( Fv/Fm with $\mathrm{Fv}=\mathrm{Fm}-\mathrm{F} 0)$ was finally calculated. Chlorophyll content was estimated in parallel on the same leaf with a handheld Chlorophyll meter (Konica Minolta SPAD-502 Plus). Values are expressed in single-photon avalanche diode units (SPAD units).

\section{Xylem sap sampling}

After $12 \mathrm{~d}$ of culture, the xylem sap of a single maize plantlet was sampled for each condition [15]. Briefly, the water potential of the third (growing) leaf was measured with a Scholander pressure chamber (M3000 model adapted with a linear slit, Soil Moisture Equipment Corp., Goleta, CA, USA). Each plantlet stem was then topped through the coleoptile, about $3 \mathrm{~cm}$ above the mesocotyl (i.e., above the starting point of nodal roots). Stump cuts were immediately washed with distilled water and 
Citation: Rozier C, Erban A, Hamzaoui J, Prigent-Combaret C, Comte G, et al. (2016) Xylem Sap Metabolite Profile Changes During Phytostimulation of Maize by the Plant Growth-Promoting Rhizobacterium, Azospirillum lipoferum CRT1. Metabolomics (Los Angel) 6: 182. doi:10.4172/21530769.1000182

blotted dry with an absorbent Whatman paper twice to remove cell debris and potential phloem sap contaminants. Stumps were then introduced in the pressure cylinder of the Scholander pressure chamber adapted with a round slit. A pressure corresponding to the measured leaf water potential was applied to the root system during $30 \mathrm{~min}$ and xylem sap was harvested with a $100 \mu \mathrm{L}$ glass syringe. Right after harvest, xylem sap was frozen in liquid nitrogen to inactivate enzymatic reactions and kept at $-80^{\circ} \mathrm{C}$ until metabolite analyses were performed.

\section{Shoot and root systems morphology analyses}

When xylem sap sampling was over, stumps were unpotted and non-adherent soil gently removed from roots with finger taps and running water. Root morphological parameters were recorded with a scanner (Expression 1680, Epson) controlled by the WinRhizo software (Regent Instruments Inc., Québec, Canada). Recorded parameters included total cumulative root length $(\mathrm{cm})$, average root diameter $(\mathrm{mm})$, number of root tips, crossings and total root surface $\left(\mathrm{cm}^{2}\right)$. Roots and shoots were then dried $\left(24 \mathrm{~h}, 110^{\circ} \mathrm{C}\right)$ and weighted.

\section{Metabolite-profiling analysis}

Seventy $\mu \mathrm{L}$ of frozen sap was thawed on ice, swiftly mixed with 10 $\mu \mathrm{L}$ of ribitol ( $1 \mathrm{mg} / \mathrm{mL}$ in methanol) and filtered through centrifugal filter units (Amicon Ultra-0.5mL $10 \mathrm{~K}$ ) to eliminate large molecular weight substances that may interfere with later silylation reactions and to remove residual enzymes that may change sap composition. Filtrates were frozen and dried in a Speed Vacuum concentrator. Ribitol was selected as an internal standard for this study according to [30,31]. Dried sap samples were mixed with $40 \mu \mathrm{L}$ of methoxyamine hydrochlorid ( 40 $\mathrm{mg} \mathrm{Ml}{ }^{-1}$ in pyridine) and incubated for $1.5 \mathrm{~h}$ at $30^{\circ} \mathrm{C}$. Then, $70 \mu \mathrm{L}$ of BSTFA (N,O-Bis(trimethylsilyl)trifluoroacetamide) and $10 \mu \mathrm{L}$ of an $\mathrm{C}_{10}, \mathrm{C}_{12}, \mathrm{C}_{15}, \mathrm{C}_{18}, \mathrm{C}_{19}, \mathrm{C}_{22}, \mathrm{C}_{28}, \mathrm{C}_{32}$, and $\mathrm{C}_{36} \mathrm{n}$-alkane mixture was added for retention index calibration [32]. The mixture was subsequently incubated for additional $30 \mathrm{~min}$ at $37^{\circ} \mathrm{C}$ to complete the reaction. Sample aliquots of 1 $\mu \mathrm{L}$ were injected in splitless mode using an Agilent 6890 injector system set to $250^{\circ} \mathrm{C}$. Initial oven temperature was maintained at $70^{\circ} \mathrm{C}$ for $1 \mathrm{~min}$, then raised to $350^{\circ} \mathrm{C}$ at $9^{\circ} \mathrm{C} \mathrm{s}^{-1}$ and kept at $350^{\circ} \mathrm{C}$ for $5 \mathrm{~min}$. All samples were run on a gas chromatography-electron impact ionization-time of flight/mass spectrometry (GC-EI-TOF/MS) instrument with an Agilent $6890 \mathrm{~N}$ gas chromatograph and a LECO Pegasus III TOF mass spectrometer using the manufacturer's ChromaTOF software for acquisition and baseline correction (versions 2.32; LECO, St. Joseph, MI, USA). Data processing and peak identity annotation was manually performed using the TagFinder visualisation tool for mass spectral matching of time groups and clusters [33]. Mass spectral features were matched to the mass spectra and retention time indices of authenticated reference metabolites from the Golm Metabolome Database, http://gmd.mpimp-golm.mpg.de/ [34]. Criteria for manually supervised metabolite annotation were the presence of at least 3 specific mass fragments per compound and a retention index deviation $<1.0 \%$ [35].

\section{Multivariate statistical analyses}

Detector responses, i.e., baseline corrected peak height of arbitrary units, were normalized by the response of the internal standard. For each compound, normalized responses were expressed as present of the maximum value obtained in the data set that consisted of 4 independent biological replicates of 4 experimental conditions, namely 2 maize cultivars either inoculated or mock-inoculated. For each biological replicate, metabolite percentages were divided by the mean value of the 4 (mock-inoculated and inoculated) plantlets of the replicate. This later step suppressed variability among biological replicates. Then, statistics were done on the normalized data to compare the 4 treatment groups using the 4 biological replicates.
All statistical analyses were carried out with the open source software R with the RV Aidememoire, mixOmics and ade4 packages (downloaded on March 2016) [36]. Non-parametric two-by-two Wilcoxon mean comparison tests were conducted to judge significances of mean differences in metabolite content between inoculated and mock-inoculated conditions for each cultivar. Significant threshold was fixed at 0.05 , but substances with a significant level above 0.1 were also recorded and quoted as tendency of difference.

\section{Results}

\section{Morphological and physiological modifications of maize root and shoot by $A$. lipoferum CRT1}

Inoculation of the maize cultivar Seiddi with A. lipoferum CRT1 had a strong effect on its root system architecture. The treatment almost doubled the root biomass of $12 \mathrm{~d}$ old plantlets (Figure 1A) caused mainly by an increase of the cumulative length of all roots (Figure 1B) with mean total root lengths of $213.7 \pm 5.5 \mathrm{~cm}$ and 280.5 $\pm 14.0 \mathrm{~cm}$ (p-value $=0.0294$, Wilcoxon test) for mock-inoculated and inoculated plants, respectively. This increase in cumulative root length was linked to a greater number of secondary roots on the radicle and lateral seminal roots, resulting in a higher number of root tips and, thus, generated an increase of total root surface. In contrast, inoculation of the maize cultivar FuturiXX had no impact on root biomass (Figure 1A) and root system architecture (Figure 1B).

Despite similar root biomass, mock-inoculated $12 \mathrm{~d}$ old plantlets of Seiddi and FuturiXX differed in shoot biomass (Figures 1A and 1C). This difference did not stem from differences in leaf length but from increased width of Seiddi leaves (data not shown). Mean photosynthetic efficiency of the youngest mature leaf, i.e., the second leaf from stem base, of the maize cultivar FuturiXX was also 35\% higher than the one of Seiddi under mock-inoculated conditions (Figure 1D). Inoculation of both cultivars with A. lipoferum CRT1 did not affect shoot biomass (Figure 1C) but enhanced the photosynthetic efficiency of the maize cultivar Seiddi by ca. $30 \%$ while leaving this parameter unaffected in the other cultivar, FuturiXX (Figure 1D). Chlorophyll content of plant leaves an indicator of nutritional status, and more specifically of nitrogen content, was estimated photometrically and yielded values that were similar for all 4 treatment groups with $29.40 \pm 4.17$ SPAD units and $35.31 \pm 1.97$ SPAD units for mock-inoculated FuturiXX and Seiddi, respectively, and $35.54 \pm 5.46$ SPAD units and $37.98 \pm 4.61$ SPAD units for inoculated FuturiXX and Seiddi [37].

\section{Impact of $A$. lipoferum CRT1 on maize leaf water potential and stem xylem sap flow}

Because xylem sap flow speed at the wound harvest site is known to affect metabolite content in the collected fluid leaf water potential that drives xylem sap upward and xylem sap harvest speed were monitored in all sampled plants [15]. As shown on Figure 2A, the water potential of the growing, and largest leaf, namely the third leaf from the stem base, was similar in all sampled plants regardless of their genotype and inoculation status. A mean water potential of $1.2 \pm 0.02$ bars was calculated $(n=16)$. Similarly, no significant difference in xylem sap harvested volumes was observed after 30 min of sap harvest among the plants we sampled, regardless of the genotype of inoculation treatment (Figure 2B), with a mean harvest volume of $2.95 \pm 0.08 \mu \mathrm{L} \cdot \mathrm{min}^{-1}$. All in all, these results demonstrate that any observed difference in metabolite contents in the harvested saps will reflect physiological differences and not sampling-related differences depending on harvest conditions. 
Citation: Rozier C, Erban A, Hamzaoui J, Prigent-Combaret C, Comte G, et al. (2016) Xylem Sap Metabolite Profile Changes During Phytostimulation of Maize by the Plant Growth-Promoting Rhizobacterium, Azospirillum lipoferum CRT1. Metabolomics (Los Angel) 6: 182. doi:10.4172/21530769.1000182
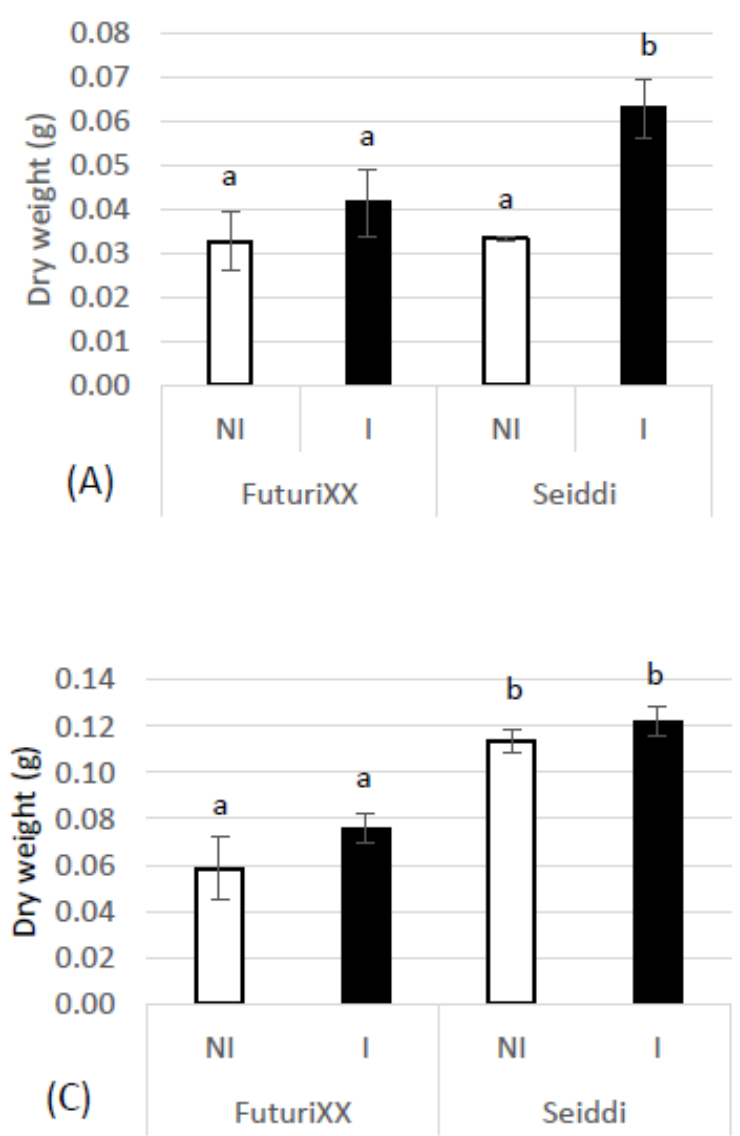
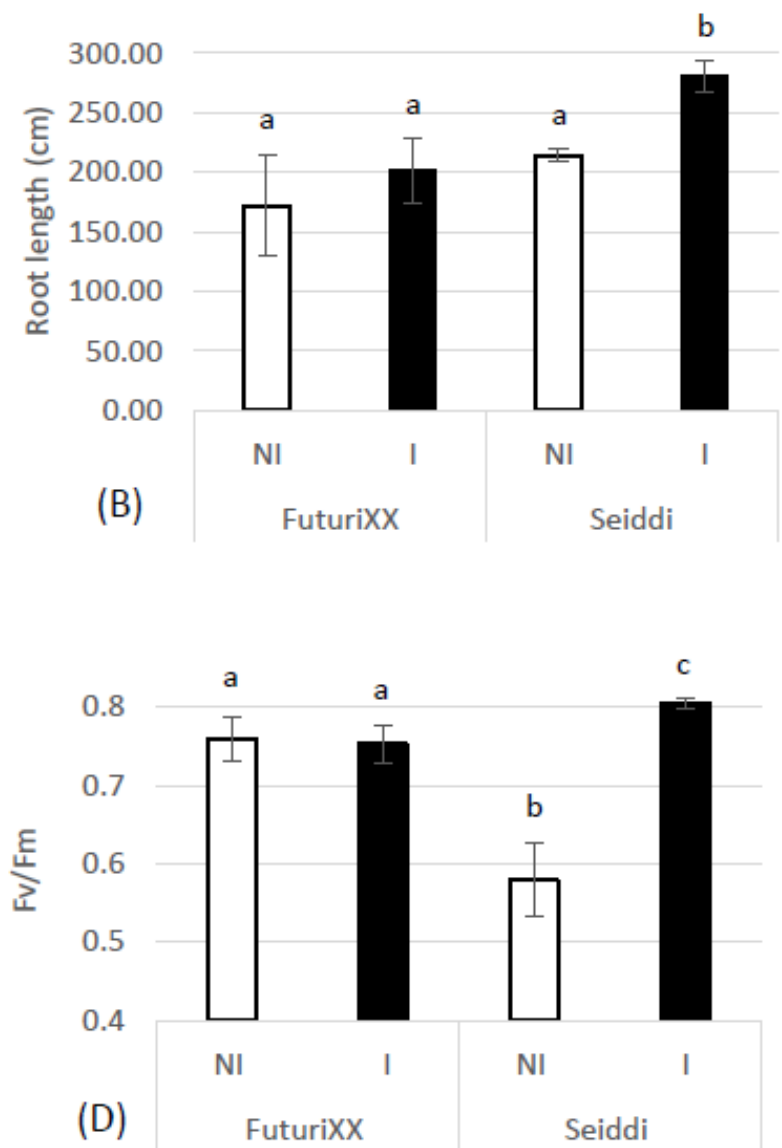

Figure 1: PGPR-induced physiological changes in maize. Root biomass (A), cumulative total root length (B), shoot biomass (C) and photosynthesis efficiency of the last mature leaf (D) were measured on two maize genotypes, FuturiXX and Seiddi, which were mock-inoculated (NI-white bars) or inoculated (I-black bars) with Azospirillum lipoferum CRT1. Results are expressed as mean \pm SE ( $n=4$ biological replicates). Statistical tests (Kruskall-Wallis non-parametric ANOVA and two-bytwo Wilcoxon tests) were conducted to determine significant differences ( $p$-value $<0.05$ ) among the 4 treatment levels and are indicated with the letters a, b and $c$.
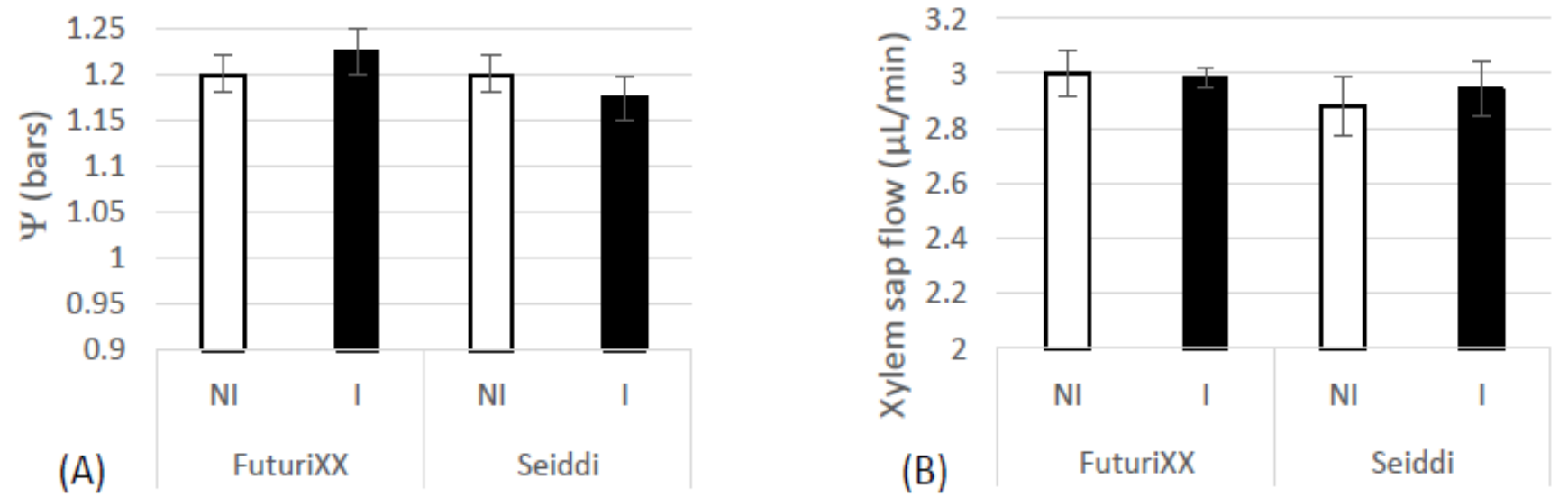

Figure 2: Third leaf water potential $(A)$ and sap harvest volume rate $(B)$ were measured on two $12 \mathrm{~d}$ old maize genotypes, FuturiXX and Seiddi, which were mockinoculated ( $\mathrm{NI}$-white bars) or inoculated (I-black bars) with Azospirillum lipoferum CRT1. Data represent means $\pm \mathrm{SE}$, $\mathrm{n}=4$ biological replicates. Non-parametric Kruskall-Wallis ANOVA did not reveal any significant difference among treatments $(p>0.05)$. 
Citation: Rozier C, Erban A, Hamzaoui J, Prigent-Combaret C, Comte G, et al. (2016) Xylem Sap Metabolite Profile Changes During Phytostimulation of Maize by the Plant Growth-Promoting Rhizobacterium, Azospirillum lipoferum CRT1. Metabolomics (Los Angel) 6: 182. doi:10.4172/21530769.1000182

Page 5 of 10

\section{Maize xylem sap metabolite content changes upon inoculation with A. lipoferum CRT1}

A total of 119 metabolites were detected among all maize xylem sap samples, including 24 linked to amino acid metabolism, 22 sugars or other carbohydrates, 6 belonging to the ascorbate or aldarate metabolism, 8 TCA cycle components, 2 urea cycle metabolites, 6 polyols, 7 phenolic compounds and 2 purine or pyrimidine metabolites (Table 1; Table S1, supplemental section). Only a subset of 104 metabolites was detected in FuturiXX, whereas all were found in Seiddi. Similarly, mock-inoculated and inoculated plantlets yielded similar sets of metabolites so that potential differences among the four treatment groups were quantitative rather than qualitative. These differences could be separated into 4 responses classes according to metabolite response patterns across all sampled xylem conditions.

The first response class contained 28 substances that specifically displayed a tendency of difference at $\mathrm{p}<0.1$, and for 15 substances significant at $\mathrm{p}<0.05$, content decreases in Seiddi xylem sap upon inoculation with A. lipoferum CRT1 (Table 1A). This group comprised carbohydrates such as glucose and its dimers sophorose and gentobiose, acids of the pentose phosphate pathway, TCA cycle or ascorbate/ aldarate metabolism, phenylpropanoids, and cadaverine. These are linked to the root growth and leaf photosynthesis-enhancing activity of the PGPR A. lipoferum CRT1.

Two other response classes grouped 28 metabolites that displayed a tendency of difference at $\mathrm{p}<0.1)$, and for 18 of them significant $(p<0.05)$, content changes that were unrelated to root growth and photosynthetic enhancement. The largest contained substances which content changes were significant in both cultivars xylem sap, and therefore represented a general response of maize to A. lipoferum CRT1 (Table 1B). This group included carbohydrates such as sucrose, maltose, fructose and mannose, the glycolytic intermediate pyruvic acid, TCA cycle intermediates, alanine and acidic amino acids and some of their metabolites, shikimate pathway metabolites, myoinositol, GABA and triethanolamine. Few metabolites were found that were decreased solely in the PGPR-irresponsive cultivar FuturiXX (only 3 if significance level was lowered to $\mathrm{p}<0.05$ (Table 1C).

Finally, 63 annotated substances displayed no significant ( $>0.1$ ) change upon inoculation with A. lipoferum CRT1 (Table S1, supplemental section) in the xylem sap of either cultivar. These included most of the detected polyols, benzoic acid derivatives, amino acid, amino acid-related metabolites, nucleotide bases and nucleotide base-related metabolites.

\section{Discussion}

\section{Azospirillum lipoferum CRT1 phytostimulatory activity on maize}

The PGPR genus Azospirillum contains more than fifteen species that modify root system architecture, stimulate shoot growth and enhance host tolerance to abiotic stresses [7]. The strain of A. lipoferum that was used in this study stimulates lateral root emergence and growth under greenhouse and European field conditions where grain and forage yields are raised $[38,39]$. The present study further reveals that lateral root growth stimulation is visible as early as $12 \mathrm{~d}$ after sowing. It occurred in the absence of visible shoot growth enhancement in agreement with earlier hypotheses that A. lipoferum stimulates shoot growth as a secondary consequence of improved nutrition caused, in part, by a more developed root system $[7,4]$. In the present study, the plants were likely at an early developmental stage and which root growth was already induced but the secondary impact on shoot growth was not yet apparent. Preceding differential growth, A. lipoferum already induced physiological changes in shoots as evidenced by enhanced photosynthetic efficiency of the youngest mature leaf.

Enhancement of leaf photosynthetic activity has been observed in numerous plant-PGPR experimental systems. For example, photosynthetic efficiency and chlorophyll content were enhanced in Arabidopsis plants inoculated with Bacillus subtilis GB03 [40]. However, most studies were conducted under drought conditions where maximum quantum efficiency $(\mathrm{Fv} / \mathrm{Fm})$ is strongly impaired and where PGPR help plants cope with their stress [3,4,41]. PGPRinduced improvements of photosystem efficiency may therefore reflect a lowering of the plant stress level. Alternatively, PGPR-mediated improvement of plant nutrition will positively impact photosynthesis by allowing leaves to produce greater amounts of photosynthetic pigments as observed in wheat plantlets inoculated with $A$. brasilense and correct impairments of photochemical conversion due to potential nutrient deficiencies [42,43]. In this study, the photosynthetic efficiency of mature maize leaves was enhanced by A. lipoferum CRT1 in the absence of any obvious abiotic stress or nutrient impairment. Indeed, soil water was not a limiting factor because it was kept at field holding capacity. Minerals may also not have been limiting because xylem sap phosphate levels and mature leaf chlorophyll contents, an indicator of leaf nitrogen content, were found to be unaffected by PGPR inoculation. In the control, PGPR-irresponsive maize cultivar, photosynthetic efficiency was also at a value that is indicative of optimum growth conditions for maize [37,44]. In conclusion, $A$. lipoferum CRT1-mediated enhancement of photosynthetic efficiency preceded growth enhancement and likely occurred independently of improved mineral nutrition or stress alleviation.

\section{Maize xylem sap content}

Currently, the most established role of xylem sap is the transfer of root-absorbed water and minerals from roots to aerial parts through what is thought to be a hollow, dead, system of xylem cells [45]. This movement is passive and requires no energy input as it is driven by a low water pressure gradient generated by water evaporation mostly at leaf stomata. Nevertheless, proteomic analyses have unveiled the presence of numerous polypeptides in xylem sap. The postulated main function of these polypeptides is to thicken and/or remodel xylem cell walls [21]. Targeted and non-targeted metabolomic analyses have established that xylem sap also contains a wide range of small, water soluble, organic substances. These include many primary metabolites such as polyols and simple sugars, amino acids and organic acids [21,22,23,26,46-50]. Xylem transported metabolites also include plant hormones $[18,20]$ and secondary metabolites that are part of the shikimate pathway and respective downstream products such as phenylpropanoids and benzoic acids $[21,23]$. The present analysis confirms the presence of most of the previously seen primary, and secondary, metabolites and expands their list in all metabolite classes with notably the organic acids glycolate, lactate and phosphate and with various primary metabolites biosynthetic intermediates. Careful elimination of contaminations and modifications through the sampling process was conducted. We could not confirm the presence of the plant hormones abscisic acid (ABA) and zeatin in the upwards directed xylem stream but we report for the first time the presence of $\gamma$-aminobutyric acid (GABA), a stress response signal over long distances in plants [51,52]. Its concentration was found to decrease in this study in agreement with the alleged stress-alleviating properties of this group of PGPR on plants [5]. The 
Citation: Rozier C, Erban A, Hamzaoui J, Prigent-Combaret C, Comte G, et al. (2016) Xylem Sap Metabolite Profile Changes During Phytostimulation of Maize by the Plant Growth-Promoting Rhizobacterium, Azospirillum lipoferum CRT1. Metabolomics (Los Angel) 6: 182. doi:10.4172/21530769.1000182

\begin{tabular}{|c|c|c|}
\hline \multirow{2}{*}{ (A) Metabolic pathways } & \multicolumn{2}{|c|}{ Metabolic variation I vs. NI (\%) } \\
\hline & FuturiXXa & Seiddia $^{a}$ \\
\hline \multicolumn{3}{|c|}{ Sugars Metabolism ${ }^{b}$} \\
\hline \multicolumn{3}{|c|}{ Sugar dimers } \\
\hline Sophorose & $7,4 \pm 14,55$ & $-42,8 \pm 4,55$ \\
\hline Gentiobiose & $1,3 \pm 3,9$ & $-37,5 \pm 7,6$ \\
\hline \multicolumn{3}{|c|}{ Galactose } \\
\hline Tagatose & $-12,8 \pm 2,2$ & $-45,2 \pm 8,2$ \\
\hline \multicolumn{3}{|c|}{ Pentose phosphate } \\
\hline Gluconic acid & $-14 \pm 9,15$ & $-39,6 \pm 5,6$ \\
\hline Glycolic acid & $-7,8 \pm 9,35$ & $-29,6 \pm 4,65$ \\
\hline \multicolumn{3}{|c|}{ Glycolysis + neoglucogenesis } \\
\hline Lactic acid & $-28,2 \pm 9,65$ & $-48,8 \pm 5,8$ \\
\hline Glucose & $-7,4 \pm 10,6$ & $-33 \pm 5,75$ \\
\hline \multicolumn{3}{|c|}{ Ascorbate and Aldarate Metabolism ${ }^{b}$} \\
\hline Arabinonic acid & $12,5 \pm 6,05$ & $-47,6 \pm 3,3$ \\
\hline Threonic acid & $15,2 \pm 19,35$ & $-31 \pm 7,55$ \\
\hline Galactaric acid & $9,7 \pm 18,95$ & $-25,3 \pm 6,8$ \\
\hline \multicolumn{3}{|c|}{ TCA Cycle $^{b}$} \\
\hline Glutaric acid, 2-oxo- & $-14,5 \pm 12,55$ & $-27,6 \pm 3,2$ \\
\hline Itaconic acid & $-14,7 \pm 12,35$ & $-25,6 \pm 7,8$ \\
\hline \multicolumn{3}{|c|}{ Amino Acids Metabolism ${ }^{b}$} \\
\hline \multicolumn{3}{|l|}{ Lysine } \\
\hline Cadaverine & $-36,1 \pm 7$ & $-43,1 \pm 5,6$ \\
\hline Lysine & $-12,3 \pm 11,05$ & $31,3 \pm 10,7$ \\
\hline \multicolumn{3}{|l|}{ Cysteine, Methionine } \\
\hline Methionine & $-5 \pm 16,15$ & $29,8 \pm 12,4$ \\
\hline \multicolumn{3}{|l|}{ Arginine, Proline } \\
\hline Ornithine-1,5-lactam & $1,3 \pm 12,65$ & $35,9 \pm 9,3$ \\
\hline \multicolumn{3}{|c|}{ Phenolic Compounds $^{b}$} \\
\hline Ferulic acid, trans- & NA & $-26,6 \pm 4,05$ \\
\hline Cinnamic acid, 4-hydroxy-, trans- & $-3,5 \pm 9,75$ & $-25,2 \pm 5,3$ \\
\hline Benzoic acid, 4-hydroxy- & NA & $-29 \pm 6,25$ \\
\hline \multicolumn{3}{|c|}{ Purine and Pyrimidine Metabolism } \\
\hline Uracil & $14,7 \pm 18,9$ & $-16,4 \pm 4,5$ \\
\hline Isopentylamine & $-16,9 \pm 8,45$ & $-36,3 \pm 10,1$ \\
\hline Butanoic acid, 2,4-dihydroxy- & $-3,1 \pm 7,5$ & $-24,8 \pm 8,95$ \\
\hline \multicolumn{3}{|c|}{ Unknown Compounds ${ }^{b}$} \\
\hline A214003 & $-6,1 \pm 9,8$ & $-30,2 \pm 3,75$ \\
\hline A213001 & $0,3 \pm 9,8$ & $-29,1 \pm 3,9$ \\
\hline A252002 & $-13,5 \pm 8,85$ & $-37,8 \pm 3,95$ \\
\hline A217004 & $3409,1 \pm 1562,75$ & $-24,6 \pm 23,25$ \\
\hline A170001 & $14,9 \pm 21,7$ & $-19,9 \pm 5,7$ \\
\hline Unknown compound-013 & $5,1 \pm 4,4$ & $-14,9 \pm 4,1$ \\
\hline \multirow{2}{*}{ (B) Metabolic pathways } & \multicolumn{2}{|c|}{ Metabolic variation I vs. $\mathrm{NI}(\%)$} \\
\hline & FuturiXXa & Seiddia \\
\hline & & \\
\hline Starch + sucrose & & \\
\hline Maltose & $-43,7 \pm 4,55$ & $-41,3 \pm 8,35$ \\
\hline Sucrose & $-11,2 \pm 2,6$ & $-10,4 \pm 2,05$ \\
\hline Fructose + mannose & & \\
\hline Mannose & $-28,2 \pm 8,25$ & $-25,4 \pm 4,8$ \\
\hline Fructose & $-15 \pm 4,3$ & $-25,1 \pm 3,6$ \\
\hline Galactose & & \\
\hline Galactinol & $-43 \pm 5,5$ & $-53,2 \pm 7,1$ \\
\hline Glycolysis + neoglucogenesis & & \\
\hline Pyruvic acid & $-28,9 \pm 2,95$ & $-40,5 \pm 2,6$ \\
\hline & & \\
\hline Malic acid & $-56,7 \pm 7,1$ & $-30,3 \pm 7,3$ \\
\hline Succinic acid & $-56,5 \pm 3,1$ & $-30,8 \pm 7$ \\
\hline
\end{tabular}


Citation: Rozier C, Erban A, Hamzaoui J, Prigent-Combaret C, Comte G, et al. (2016) Xylem Sap Metabolite Profile Changes During Phytostimulation of Maize by the Plant Growth-Promoting Rhizobacterium, Azospirillum lipoferum CRT1. Metabolomics (Los Angel) 6: 182. doi:10.4172/21530769.1000182

Page 7 of 10

\begin{tabular}{|c|c|c|}
\hline \multicolumn{3}{|c|}{ Amino Acids Metabolism ${ }^{b}$} \\
\hline \multicolumn{3}{|l|}{ Alanine, Aspartic acid, Glutamate } \\
\hline Butanoic acid, 4-amino- & $-30 \pm 6,75$ & $-26 \pm 3,95$ \\
\hline Aspartic acid & $-28,5 \pm 7,7$ & $-20 \pm 2,95$ \\
\hline Alanine & $-23,1 \pm 7,75$ & $-15,8 \pm 3,8$ \\
\hline Glutamic acid & $-24,5 \pm 5,65$ & $-18,3 \pm 4,55$ \\
\hline \multicolumn{3}{|l|}{ Cysteine, Methionine } \\
\hline Serine, O-acetyl- & $-18,4 \pm 3,95$ & $31,5 \pm 6,65$ \\
\hline \multicolumn{3}{|l|}{ Aromatic amino acids } \\
\hline Shikimic acid & $-43,9 \pm 5$ & $-37,5 \pm 4,4$ \\
\hline Quinic acid & $-44,2 \pm 5,15$ & $-35,2 \pm 6$ \\
\hline \multicolumn{3}{|c|}{ Polyols $^{b}$} \\
\hline Inositol, myo- & $-26,4 \pm 4,9$ & $-10,9 \pm 4,25$ \\
\hline \multicolumn{3}{|c|}{ Other Pathway Metabolites ${ }^{b}$} \\
\hline Triethanolamine & $-24,4 \pm 1,85$ & $-64,3 \pm 4,4$ \\
\hline \multirow{2}{*}{ (C) Metabolic pathways } & \multicolumn{2}{|c|}{ Metabolic variation I vs. NI (\%) } \\
\hline & FuturiXXa & Seiddia \\
\hline \multicolumn{3}{|c|}{ TCA Cycle ${ }^{\mathrm{b}}$} \\
\hline Aconitic acid, cis- & $-34,5 \pm 12,75$ & $-18,9 \pm 13,75$ \\
\hline Fumaric acid & $-40,6 \pm 12,35$ & $-17,9 \pm 8,05$ \\
\hline \multicolumn{3}{|c|}{ Amino Acids Metabolismb } \\
\hline \multicolumn{3}{|c|}{ Alanine, Aspartic acid, Glutamate } \\
\hline Pyroglutamic Acid + Glutamic Acid + Glutamine & $-20,2 \pm 5,25$ & $-1,9 \pm 11,7$ \\
\hline \multicolumn{3}{|l|}{ Glycine, Serine, Threonine } \\
\hline Glycine & $-8 \pm 1,25$ & $-16,6 \pm 5,4$ \\
\hline \multicolumn{3}{|c|}{ Urea Cycle Metabolism ${ }^{b}$} \\
\hline Urea & $-15,1 \pm 3$ & $19,1 \pm 21,2$ \\
\hline \multicolumn{3}{|c|}{ Other Pathway Metabolites ${ }^{b}$} \\
\hline Glycyl-phenylalanine & $20,5 \pm 6,1$ & $12,6 \pm 13,1$ \\
\hline \multicolumn{3}{|c|}{ Unknown Compounds ${ }^{b}$} \\
\hline similar to Ditertbutylphenol & $11,4 \pm 2,2$ & $4 \pm 2,35$ \\
\hline A168011 & $-18 \pm 8,5$ & $22,7 \pm 27,9$ \\
\hline A237001 & $-20,7 \pm 7,4$ & $-22,1 \pm 8,7$ \\
\hline A167004 & $-16,3 \pm 3,5$ & $15,9 \pm 19,15$ \\
\hline A311002 & $-33,4 \pm 6,8$ & $-15 \pm 14,7$ \\
\hline
\end{tabular}

${ }^{a}$ Ratios of mean values of inoculated plantlets over mock-inoculated plantlets were expressed as percentage values \pm relative standard error. Wilcoxon non-parametric tests were conducted for each metabolite to compare inoculated and non-inoculated mean values in each cultivar. They are indicated as a background color (Dark grey, $\mathrm{p}<0.05$; light grey, $\mathrm{p}<0.1$; and white, $\mathrm{p}>0.1$ ). NA indicates that the metabolite was detected in less than 2 of biological replicate samples. ${ }^{\mathrm{b}} \mathrm{Metabolites}$ were classified according to KEGG's metabolic pathway database and grouped as (A) metabolites that with $p<0.1$ in Seiddi, (B) both cultivars or (C) FuturiXX. Metabolites unaffected by $A$. lipoferum CRT1 inoculation are shown in Table S1 supplemental section. Yet non-identified samples are indicated by the code of the Golm Metabolome Database.

Table 1: Percent variation of metabolite contents in the xylem sap of FuturiXX and Seiddi maize genotypes upon $A$. lipoferum CRT1 inoculation.

number of detected hormones is small given the predicted importance of xylem sap as a vehicle for root-to-shoot communication in plants, indicating that current analyses are still biased by analytical limitations, such as insufficient sensitivity and/or the fact that many hormones may be transported as conjugated forms that are not yet present in databases to allow their identification [52]. Greater effort in this area is therefore strongly needed.

Because xylem cells are not considered as metabolically active, it is likely to assume that controlled secretion and uptake by neighbouring parenchyma, protoxylem and/or sugar-rich phloem cells of the root system will enrich, or deplete, xylem sap of specific metabolites and subsequently on the path to the shoot apex (Shi et al. [45]). Xylem sap composition is, therefore, expected to reflect metabolite flux directions within the root though functional analyses of organic metabolite transporters in plant roots are so far limited.

\section{Primary metabolites as a signature of the root status}

Previous studies with the same PGPR strain have revealed that it expresses its phytostimulatory activity in a cultivar-specific manner in maize, a property shared with other PGPR genera $[26,29,53]$. Through qPCR analyses, these studies have shown that bacterial colonisation of the root system occurs at similar level, irrespective of its growth response. In agreement, this study further reveals that A. lipoferum triggers metabolite content decreases as a generic response of maizeAzospirillum interaction. These were mostly simple sugars, amino acids and intermediates of starch degradation, glycolysis and TCA cycle in line with the loss of similar metabolites by roots as food contribution to their PGPR partner as exudates [54]. It is therefore hypothesized that PGPR-mediated increased organic matter exudation increases the flux of a variety of energetic metabolites towards the outside of the root at the expense of its xylem sap interior.

Additional metabolites displayed content decreases solely in the phytostimulated maize genotype. These were glucose, some of its low concentrated dimers, acidic derivatives of pentose phosphate and ascorbate/aldarate metabolisms, cadaverin and lactic acid. Their increased uptake from the xylem sap by neighbouring cell types may have been caused by PGPR-induced lateral root growth to sustain the increased metabolic needs of dividing cells with for, notably, the de 
Citation: Rozier C, Erban A, Hamzaoui J, Prigent-Combaret C, Comte G, et al. (2016) Xylem Sap Metabolite Profile Changes During Phytostimulation of Maize by the Plant Growth-Promoting Rhizobacterium, Azospirillum lipoferum CRT1. Metabolomics (Los Angel) 6: 182. doi:10.4172/21530769.1000182

Page 8 of 10

novo synthesis of cell wall polymers like cellulose that is solely made of glucose. Although increases in exudation and cell division will both lead to the uptake of metabolites from xylem sap, these two processes may not affect similar metabolite transporters therefore explaining that they decrease the content of different sets of metabolites.

Because xylem sap moves up to the shoot, any single of its metabolites, or any pattern of multiple primary metabolites may act as a metabolic signature to retrograde signal the metabolic status and needs of the roots (sink) to the aerial (source) parts of the plant. In concordance with the current knowledge on glucose signalling and growth inhibition, a plausible metabolic candidate signal could be glucose [55-58]. Glucose is the end product of photosynthesis transported via the phloem transport metabolite sucrose to the root system and thereby the main carbon supply and starting substrate of energy metabolism for both root and PGPR growth. However, independently of its energetic value, glucose constitutes one of the major cellular and long distance developmental signals that ensures that engagement into irreversible growth is matched by adequate supply of energy-rich $\mathrm{C}$-substance and that sugar production by photosynthesis is in phase with sugar store level targets [33]. Glucose is indeed recognized by various plant cell membrane-bound and cytoplasmic proteins such as Hexokinase (HXK) that modulate the transcription of many developmental genes including some coding for biosynthetic enzymes and signalling elements of most developmental hormones such as auxins, cytokinins, gibberellins, abscisic acid and brassinosteroids [57]. Nevertheless, glucose and growth hormone signalling networks are intertwined in a way that is neither direct nor hierarchical so that it is equally possible that Azospirillum-induced lowered glucose levels affects hormonal signalling to stimulate growth or that Azospirillum-produced auxins affect plant growth and consequently glucose level in the xylem [55,58]. Additionally, glucose, as one of the primary photosynthesis products, is known to exert an inhibitory activity on photosystems photochemical conversion efficiency through direct Calvin-cycle enzyme inhibition, even from an extracellular location $[59,60]$. It is therefore also hypothesized that PGPR-induced xylem sap glucose content lowering led to the observed increased photosynthetic potential to enhance C-sequestration and sustain increased root biomass formation. In agreement with a proposed role of xylem sap glucose in PGPR-modulated root growth signalling and leaf photosynthesis, glucose content, and not the one of the other hexose isomers, fructose and mannose, nor of the sugar dimers maltose and saccharose, was differentially lowered in the two cultivars of this study following the Azospirillum-induced phytostimulatory response in maize. In a separate study lower glucose content and increased photosynthesis activity were observed in Arabidopsis leaves after root inoculation with the PGPR Bacillus subtilis [40].

\section{Secondary metabolite signature}

Xylem sap phenolic compounds also exhibited an interesting response signature after Azospirillum inoculation. Early biosynthetic metabolites belonging to the shikimate pathway, namely shikimic acid and quinic acid, decreased in content as a generic response to maizeAzospirillum interaction. Nevertheless, most downstream products of the shikimate pathway such as aromatic amino acids, phenolic acid and coumarin derivatives were unaffected by Azospirillum inoculation. Only phenylpropanoids were found to specifically respond in the phytostimulated maize cultivar. Because phenylpropanoids serve as building blocks for the in situ synthesis of monolignols and later deposition as lignin polymer in the sclerenchymatous xylem cell walls (Ferrer etal. [61]), the results of this study suggest that phytostimulation of maize by Azospirillum may modify the lignin content of the host plant. Such hypothesis agrees with earlier IR spectrum analyses that demonstrated modifications of the lignin over cellulose ratio in maize cell walls after A. lipoferum inoculation [39,61]. The fact that previous studies have shown enhancements of phenylpropanoids, or phenylpropanoid products, in both maize and rice roots after $A$. lipoferum inoculation suggests that sequestration of phenylproponoids by roots may be the cause of the decrease of shikimic acid and quinic acid in retrograde transported xylem sap $[62,63]$.

\section{Acknowledgements}

Elise Lacroix and the greenhouse technical platform of FR BioEnviS (University Lyon 1) are thanked for plant caring and harvest.

Funding CR was supported by a fellowship from the "Ministère de l'enseignement supérieur et de la recherche " and the work was funded by the national research agency of France (ANR), project ANR-12-AGRO-

\section{References}

1. Tahir M, Sarwar MA (2013) Plant growth promoting rhizobacteria (PGPR): A budding complement of synthetic fertilizers for improving crop production. Pak $J$ Life Soc Sci Pak

2. Martínez-Viveros $O$, Jorquera MA, Crowley DE, Gajardo G, Mora ML, et al (2010) Mechanisms and practical considerations involved in plant growth promotion by rhizobacteria. J Soil Sci Plant Nutr 10: 293-319.

3. Drogue B, Doré H, Borland S, Wisniewski-Dyé F, Prigent-Combaret C (2012) Which specificity in cooperation between phytostimulating rhizobacteria and plants? Res Microbiol 163: 500-510.

4. Vacheron J, Desbrosses G, Bouffaud ML, Touraine B, Moënne-Loccoz Y, et al. (2013) Plant growth-promoting rhizobacteria and root system functioning Front Plant Sci 4: 356.

5. Wisniewski-Dyé FB, Drogue S, Borland C, Prigent-Combaret (2012) Azospirillumplant interaction: from root colonization to plant growth promotion. In Beneficial Plant-Microbe Interactions: Ecology and Applications, pp: 237-269.

6. Veresoglou SD, Menexes G (2010) Impact of inoculation with Azospirillum spp. on growth properties and seed yield of wheat: a meta-analysis of studies in the ISI Web of Science (1981-2008). Plant Soil 337: 469-480.

7. Bashan Y, Holguin G, de-Bashan LE (2004) Azospirillum-plant relationships: physiological, molecular, agricultural, and environmental advances (19972003). Can J Microbiol 50: 521-577.

8. Gururani MA, Upadhyaya CP, Baskar V, Venkatesh J, Nookaraju A, et al. (2012) Plant Growth-Promoting Rhizobacteria Enhance Abiotic Stress Tolerance in Solanum tuberosum Through Inducing Changes in the Expression of ROS-Scavenging Enzymes and Improved Photosynthetic Performance. Jou Pla Grow Regul 32: 245-258.

9. Weller DM, Mavrodi DV, van Pelt JA, Pieterse CMJ, van Loon LC, et al. (2012) Induced Systemic Resistance in Arabidopsis thaliana Against Pseudomonas syringae pv tomato by 2,4-Diacetylphloroglucinol-Producing Pseudomonas fluorescens. Phytopathol 102: 403-412.

10. Kuc J (2001) Concepts and Direction of Induced Systemic Resistance in Plants and its Application. Eur Jour Plant Pathol 107: 7-12.

11. Davies WJ, Zhang J (1991) Root Signals and the Regulation of Growth and Development of Plants in Drying Soil. Annu Rev Plant Physiol Plant Mol Biol 42: 55-76.

12. Dodd IC (2005) Root-to-shoot signalling: Assessing the roles of "up" in the up and down world of long-distance signalling in planta. In: Lambers $\mathrm{H}$, Colmer TD (eds.) Root Physiology: From Gene to Function, pp: 251-270.

13. Thieme CJ, Rojas-Triana M, Stecyk E1, Schudoma C1, Zhang W1, et al. (2015) Endogenous Arabidopsis messenger RNAs transported to distant tissues. Nat Plants 1: 15-25.

14. Jackson M (1997) Hormones from roots as signals for the shoots of stressed plants. Trends Plant Sci 2: 22-28.

15. Goodger JQ, Sharp RE, Marsh EL, Schachtman DP (2005) Relationships between xylem sap constituents and leaf conductance of well-watered and water-stressed maize across three xylem sap sampling techniques. J Exp Bot 56: $2389-2400$. 
Citation: Rozier C, Erban A, Hamzaoui J, Prigent-Combaret C, Comte G, et al. (2016) Xylem Sap Metabolite Profile Changes During Phytostimulation of Maize by the Plant Growth-Promoting Rhizobacterium, Azospirillum lipoferum CRT1. Metabolomics (Los Angel) 6: 182. doi:10.4172/21530769.1000182

16. Liang J, Zhang J (1997) Collection of Xylem Sap at Flow Rate Similar to in vivo Transpiration Flux. Plant Cell Physiol 38: 1375-1381.

17. Bahrun A, Jensen CR, Asch F, Mogensen VO (2002) Drought-induced changes in xylem $\mathrm{pH}$, ionic composition, and ABA concentration act as early signals in field-grown maize (Zea mays L.). J Exp Bot 53: 251-263.

18. Dong H, Niu Y, Li W, Zhang D (2008) Effects of cotton rootstock on endogenous cytokinins and abscisic acid in xylem sap and leaves in relation to lea senescence. J Exp Bot 59: 1295-1304.

19. Korovetska H, Novák O, Juza O, Gloser V (2014) Signalling mechanisms involved in the response of two varieties of Humulus lupulus $L$. to soil drying: changes in xylem sap $\mathrm{pH}$ and the concentrations of abscisic acid and anions. Plant Soil 380: 375-387.

20. Zhang J, Davies WJ (1990) Changes in the concentration of ABA in xylem sap as a function of changing soil water status can account for changes in leaf conductance and growth. Plant Cell Environ 13: 277-285

21. Alvarez S, Marsh EL, Schroeder SG, Schachtman DP (2008) Metabolomic and proteomic changes in the xylem sap of maize under drought. Plant Cell Environ 31: $325-340$.

22. Sung J, Sonn Y, Lee Y, Kang S, Ha S, et al. (2015) Compositional changes of selected amino acids, organic acids, and soluble sugars in the xylem sap of $\mathrm{N}$, P, or K-deficient tomato plants. Jour Plant Nutr Soil Sci 178: 792-797.

23. Rellán-Álvarez R, El-Jendoubi H, Wohlgemuth G, Abadía A, Fiehn O, et al. (2011) Metabolite profile changes in xylem sap and leaf extracts of strategy plants in response to iron deficiency and resupply. Front Plant Sci 2: 66.

24. Kopka J (2006) Current challenges and developments in GC-MS based metabolite profiling technology. J Biotechnol 124: 312-322.

25. Fages J, Mulard D (1988) Isolement de bactéries rhizosphériques et effet de leur inoculation en pots chez Zea mays. Agronomie 8: 309-314.

26. Walker V, Bertrand, C, Bellvert, F, Moënne-Loccoz Y, Bally R, et al. (2011) Host plant secondary metabolite profiling shows a complex, strain-dependent response of maize to plant growth-promoting rhizobacteria of the genus Azospirillum. New Phytol 189: 494-506.

27. Nelson LM, Knowles R (1978) Effect of oxygen and nitrate on nitrogen fixation and denitrification by Azospirillum brasilense grown in continuous culture. Can $\mathrm{J}$ Microbiol 24: 1395-1403.

28. Sambrook GA (1990) Molecular cloning: A laboratory manual. ( $2^{\text {nd }} E d n$.) Current protocols in molecular biology. Cell 61: 17-18.

29. Couillerot O, Bouffaud ML, Baudoin E, Muller D (2010) Development of a realtime PCR method to quantify the PGPR strain Azospirillum lipoferum CRT1 on maize seedlings. Soil Biol Biochem 42: 2298-2305.

30. Lisec J, Schauer N, Kopka J, Willmitzer L, Fernie AR (2006) Gas chromatography mass spectrometry-based metabolite profiling in plants. Nat Protoc 1: 387-396.

31. Ribeiro PR, Fernandez LG, de Castro RD, Ligterink W, Hilhorst HW (2014) Physiological and biochemical responses of Ricinus communis seedlings to different temperatures: a metabolomics approach. BMC Plant Biol 14: 223.

32. Erban A, Schauer N, Fernie A, Kopka J (2007) Nonsupervised Construction and Application of Mass Spectral and Retention Time Index Libraries From Time-of-Flight Gas Chromatography-Mass Spectrometry Metabolite Profiles. In Metabolomics, Weckwerth W (eds) pp: 19-38.

33. Luedemann A, Strassburg K, Erban A, Kopka J (2008) TagFinder for the quantitative analysis of gas chromatography--mass spectrometry (GC-MS)based metabolite profiling experiments. Bioinformatics 24: 732-737.

34. Schauer N, Steinhauser D, Strelkov S, Schomburg D, Allison G, et al. (2005) GC-MS libraries for the rapid identification of metabolites in complex biological samples. FEBS Lett 579: 1332-1337.

35. Strehmel N, Hummel J, Erban A, Strassburg K, Kopka J (2008) Retention index thresholds for compound matching in GC-MS metabolite profiling. J Chromatogr B Analyt Technol Biomed Life Sci 871: 182-190.

36. R Development Core Team (2008) R: A language and environment for statistical computing.

37. Piekielek WP, Fox RH, Toth JD, Macneal KE (1995) Use of a Chlorophyll Meter at the Early Dent Stage of Corn to Evaluate Nitrogen Sufficiency. Agron 87: 403-408.

38. E Zemrany H, Czarnes S, Hallett PD, Alamercery S, Bally R, et al. (2007) Early changes in root characteristics of maize (Zea mays) following seed inoculation with the PGPR Azospirillum lipoferum CRT1. Plant Soil 291: 109-118.
39. E Zemrany H, Cortet J, Peter Lutz M, Chabert A, Baudoin E, et al. (2006) Field survival of the phytostimulator Azospirillum lipoferum CRT1 and functional impact on maize crop, biodegradation of crop residues, and soil fauna indicators in a context of decreasing nitrogen fertilisation. Soil Biol Biochem 38: $1712-1726$.

40. Zhang H, Xie X, Kim MS, Kornyeyev DA, Holaday S, et al. (2008) Soil bacteria augment Arabidopsis photosynthesis by decreasing glucose sensing and abscisic acid levels in planta. Plant J 56: 264-273.

41. Silva MA, Jifon, JL, Silva JAG, Sharma V (2007) Use of physiological parameters as fast tools to screen for drought tolerance in sugarcane. Braz $J$ Plant Physiol 19: 193-201.

42. Bashan Y, Bustillos JJ, Leyva LA, Hernandez JP, Bacilio M (2006) Increase in auxiliary photoprotective photosynthetic pigments in wheat seedlings induced by Azospirillum brasilense. Biol Fertil Soils 42: 279-285.

43. Beardall J, Young E, Roberts S (2001) Approaches for determining phytoplankton nutrient limitation. Aquat Sci 63: 44-69.

44. Liu YF, Qi MF, Li TL (2012) Photosynthesis, photoinhibition, and antioxidant system in tomato leaves stressed by low night temperature and their subsequent recovery. Plant Sci 196: 8-17.

45. Shi H, Quintero FJ, Pardo JM, Zhu JK (2002) The putative plasma membrane $\mathrm{Na}(+) / \mathrm{H}(+)$ antiporter SOS1 controls long-distance $\mathrm{Na}(+)$ transport in plants. Plant Cell 14: 465-477.

46. Iwai H, Usui M, Hoshino H, Kamada H, Matsunaga T, et al. (2003) Analysis of sugars in squash xylem sap. Plant Cell Physiol 44: 582-587.

47. Krishnan HB, Natarajan SS, Bennett JO, Sicher RC (2011) Protein and metabolite composition of xylem sap from field-grown soybeans (Glycine max). Planta 233: 921-931.

48. Oda A, Shimizu M, Kuroha T, Satoh S (2005) Induction of xylem sap methylglycine by a drought and rewatering treatment and its inhibitory effects on the growth and development of plant organs. Physiol Plant 124: 515-523.

49. Tatár E, Mihucz VG, Kmethy B, Záray G, Fodor F (2000) Determination of organic acids and their role in nickel transport within cucumber plants. Microchem J 67: 73-81.

50. Wei Z, Wong JW, Hong F, Zhao H, Li H, et al. (2007) Determination of inorganic and organic anions in xylem saps of two contrasting oilseed rape varieties: Roles of anions in long-distance transport of cadmium. Microchem J 86: 53-59.

51. Nayyar H, Kaur R, Kaur S, Singh R (2013) Aminobutyric Acid (GABA) Imparts Partial Protection from Heat Stress Injury to Rice Seedlings by Improving Leaf Turgor and Upregulating Osmoprotectants and Antioxidants. J Plant Growth Regul 33: 408-419.

52. Piotrowska A, Bajguz A (2011) Conjugates of abscisic acid, brassinosteroids ethylene, gibberellins, and jasmonates. Phytochemistry 72: 2097-2112.

53. Arsac JF, Lamothe C, Mulard D, Fages J (1990) Growth enhancement of maize (Zea mays $L$ ) through Azospirillum lipoferum inoculation: effect of plant genotype and bacterial concentration. Agron Sustain Dev 10: 649-654.

54. Phillips DA, Fox TC, King MD, Bhuvaneswari TV, Teuber LR (2004) Microbia products trigger amino acid exudation from plant roots. Plant Physiol 136 2887-2894.

55. Cheng WH, Endo A, Zhou L, Penney J, Chen HC, et al. (2002) A unique shortchain dehydrogenase/reductase in Arabidopsis glucose signaling and abscisic acid biosynthesis and functions. Plant Cell 14: 2723-2743.

56. Cho JI, Ryoo N, Eom JS, Lee DW, Kim HB, et al. (2009) Role of the rice hexokinases OsHXK5 and OsHXK6 as glucose sensors. Plant Physiol 149 745-759.

57. Ljung K, Nemhauser JL2, Perata P3 (2015) New mechanistic links between sugar and hormone signalling networks. Curr Opin Plant Biol 25: 130-137.

58. Yanagisawa S, Yoo SD, Sheen J (2003) Differential regulation of EIN3 stability by glucose and ethylene signalling in plants. Nature 425: 521-525.

59. Goldschmidt EE, Huber SC (1992) Regulation of photosynthesis by endproduct accumulation in leaves of plants storing starch, sucrose, and hexose sugars. Plant Physiol 99: 1443-1448. 
Citation: Rozier C, Erban A, Hamzaoui J, Prigent-Combaret C, Comte G, et al. (2016) Xylem Sap Metabolite Profile Changes During Phytostimulation of Maize by the Plant Growth-Promoting Rhizobacterium, Azospirillum lipoferum CRT1. Metabolomics (Los Angel) 6: 182. doi:10.4172/21530769.1000182

60. Stitt, M, Schaewen A, Willmitzer L (1991) "Sink" regulation of photosynthetic metabolism in transgenic tobacco plants expressing yeast invertase in their cell wall involves a decrease of the Calvin-cycle enzymes and an increase of glycolytic enzymes. Planta 183: 40-50.

61. Ferrer JL, Austin MB, Stewart C Jr, Noel JP (2008) Structure and function of enzymes involved in the biosynthesis of phenylpropanoids. Plant Physiol Biochem 46: 356-370.
62. Walker V, Couillerot O, Felten A, Bellvert F, Jansa J, et al. (2011) Variation of secondary metabolite levels in maize seedling roots induced by inoculation with Azospirillum, Pseudomonas and Glomus consortium under field conditions. Plant Soi 356: 151-163.

63. Chamam A, Sanguin H, Bellvert F, Meiffren G, Comte G, et al. (2013) Plant secondary metabolite profiling evidences strain-dependent effect in the Azospirillum-Oryza sativa association. Phytochemis 87: 65-77. 